\title{
Should We Be Concerned? Comparison of Catheter and CT Angiogram for Arterial Distensibility at the Site of Carotid Stenosis during Catheter Angiography
}

\author{
Aly Abayazeed, Juan Diego Lozano, Gabriela Santos-Nunez*, Srinivasan Vendantham, Ajit Puri, \\ Ajay Wakhloo, Deepak Takhtani
}

Department of Radiology, University of Massachusetts Medical School, Worcester, MA, USA

Email: *gabriela.santos-nunez@umassmemorial.org, Aly.Abayazeed@umassmemorial.org, jd.lozano@providence.org, svendantham@radiology.arizona.edu,Ajit.Puri@umassmemorial.org,Ajay.Wakhloo@umassmemorial.org, deepak.takhtani@umassmemorial.org

How to cite this paper: Abayazeed, A., Lozano, J.D., Santos-Nunez, G., Vendantham, S., Puri, A., Wakhloo, A. and Takhtani, D. (2017) Should We Be Concerned? Comparison of Catheter and CT Angiogram for Arterial Distensibility at the Site of Carotid Stenosis during Catheter Angiography. Open Journal of Medical Imaging, 7, 237-247.

https://doi.org/10.4236/ojmi.2017.74022

Received: September 6, 2017

Accepted: November 27, 2017

Published: November 30, 2017

Copyright ( 92017 by authors and Scientific Research Publishing Inc. This work is licensed under the Creative Commons Attribution International License (CC BY 4.0).

http://creativecommons.org/licenses/by/4.0/

(c) (i) Open Access

\begin{abstract}
Purpose: During catheter angiogram (CA) there is momentary increase in intravascular volume and pressure due to intra-arterial injection that can potentially cause vascular distention at the stenotic site, whereas on CT angiogram (CTA) is unlikely due to intravenous administration. Methods: CA and CTA of the carotid artery from 29 patients were retrospectively studied. CA and CTA were obtained for each patient. Curved sagittal MPRs mirroring the carotid artery on CA were used to measure the diameter at stenosis and at the distal lumen. Mural plaque calcium content was graded on axial CTAs. Results: Accounting for repeated measurements, the likelihood that the lumen diameter from CA will be larger than CTA was higher at stenosis than distal to it but the difference in lumen diameters at stenosis was similar to CTA. There is insufficient evidence that intra-arterial hand-injection during CA leads to underestimation of the degree of stenosis. Percentage stenosis using the NASCET criteria differed between the 3 measurements, post hoc analysis showed significant difference between CA and axial CTA $(p<0.0001)$, but not between CA and curved sagittal CTA $(p>0.99)$. The difference in lumen diameter did not depend on our calcium grading $(p=0.484)$. Conclusions: There is insufficient evidence to suggest that intra-arterial hand-injection contributes to vessel distention and underestimation of percent stenosis during CA in this study. Mural plaque calcium does not affect the degree of stenosis on CTA.
\end{abstract}

\section{Keywords}

Arterial Distensibility, Carotid Stenosis, Catheter Angiogram 


\section{Introduction}

Decreased arterial distensibility of a diseased vessel as a risk factor for coronary vascular disease (CVD) is well studied in the cardiology literature [1]. Functional impairment of the arterial wall may occur earlier than the structural abnormalities of the vessel wall [2]. The same observations applied to the coronary arteries can be applied to the carotid arteries in the neck. The decreased carotid distensibility in carotid atherosclerotic disease is expected to affect the blood flow and blood volume under normal physiologic condition, which is the case on CT angiography. However, the iatrogenic hand injection of contrast material on conventional angiography is expected to distend the vessel beyond what is expected under physiologic conditions secondary to the increase in pressure according to the Hagen-Poiseuille equation $\left(Q=\Delta P \cdot \pi \cdot r^{4} / 8 \mu L\right.$, where $Q=$ Flow, $\Delta P=$ Pressure loss, $\pi=$ Mathematical constant $\mathrm{Pi}, r=$ Radius of pipe, $\mu=$ Dynamic viscosity and $L=$ Length of pipe) [3]. A number of publications compared the percent carotid stenosis between CTA and CA, quantified the degree of stenosis according to the NASCET criteria on CTA and evaluated the relation between the volume of calcification in the mural plaques and the degree of vascular stenosis [4] [5] [6] [7]. Nevertheless, to our knowledge this is the first literature work evaluating the exact relationship between CTA and CA measurement at the stenotic segment hypothesizing distensibility induced underestimation of the degree of stenosis secondary to hand injection induced vascular distensibility on CA.

\section{Materials and Methods}

\subsection{Inclusion Criteria and Imaging}

Examinations were retrospectively collected from a single institution from $\mathrm{Au}$ gust 2010 through October 2014 after IRB approval and HIPAA compliance; no patient consent was required. Examinations from 29 patients (16 males, $13 \mathrm{fe}-$ males, median age of 65) with known carotid artery stenotic disease that received CT angiography studies followed by CA were entered into the study. Examinations were not included if the purpose of imaging was due to trauma, dissection, vascular anomaly/malformation, tumor evaluation, or primary evaluation of the posterior circulation. Examinations were excluded because of inadequate coverage and/or technical errors precluding full evaluation of the cervical carotid arteries.

GE and Philips Medical Systems were used for the CTA examinations. The CTA images were obtained from the C6 level to skull vertex by helical HS mode at $0.9 \mathrm{~mm}$ slice thickness, 0.5 pitch, $22 \mathrm{~cm}$ FOV and $512 \times 512$ matrix and $0.43 \mathrm{~mm}$ voxels $(120 \mathrm{kVp}, 350 \mathrm{~mA})$. Intravenous access was via an antecubital vein by using an 18-or 20-gauge angiocatheter. A total of $80 \mathrm{~mL}$ Isovue 350 were injected at a rate of 4.0 to $4.5 \mathrm{~mL} / \mathrm{s}$, with a 17 -second delay or the use of Smart Prep at the pulmonary artery. CT technologists performed all the post-processing multiplanar reformats (MPRs) at the CT operator's console. Coronal and sagittal MPR images were created as $10.0 \mathrm{~mm}$ thick, spaced by 3 
$\mathrm{mm}$. In some cases, rotational MPRs were created at the carotid bifurcations with a thickness of $7 \mathrm{~mm}$ and spacing by $3 \mathrm{~mm}$. Additionally the evaluating radiologist performed curved sagittal $0.45 \mathrm{~mm}$ MPRs mirroring the carotid artery on CA (VOXAR-3D) at workstation. 3D-rendered images were created on a GE or Philips workstation. All images were viewed on IDX-IMAGECAST Philips PACS workstations.

A Philips medical system (Allura Xper FD20/15) was used for the CA examinations. Two orthogonal views were obtained with $8 \mathrm{cc}$ hand injection of Isovue 250 at approximately $5 \mathrm{cc} / \mathrm{s}$. CA images were auto calibrated for magnification. All images were viewed on IDX-IMAGECAST Philips PACS workstations.

\subsection{Evaluation of Cases}

Diameter of carotid stenosis on CTA versus carotid diameter on CA were the considered variables. Measurement of carotid stenosis was at the narrowest portion of the carotid bulb on the axial source image and the curved sagittal thin section MPR images created by the radiologist. The reformatted thin slice curved sagittal images were specifically chosen because the lumen measurement correlated best with CA and the vessel orientation was created to mirror the CA view. Despite, that the curved sagittal MPRs suffer from data interpolation that could potentially result in overestimation of the vessel size secondary to edge blur, the stenosis ratio as per NASCET correlated better with the CA numbers than axial CTA which does not suffer from this problem [8]. The use of axial versus sagittal CTA images to evaluate the degree of carotid stenosis has been a subject of debate, however, we consistently obtained very similar percentages of stenosis when comparing the reformatted thin slice curved sagittal images, that were created to mirror the carotid artery orientation on CA, and the CA images. We recommend using these reformats whenever possible (Figure 1). Two independent radiology fellows evaluated the cases. MPRs were used to identify the carotid orientation to ensure true cross-sectional measurements. The internal carotid arteries (ICAs) identified as passing oblique to the axial plane were measured perpendicular to their own oblique axis as seen on the MPRs. The distal ICA was measured well past the bulb where the walls are parallel and no longer tapering from the carotid bulb as per NASCET [9] [10]. Millimeter measurements were obtained by using the sub-millimeter measurement and magnification tools on the PACS workstation.

Special attention was directed to some of the more densely calcified plaques, with extra windowing used when needed to better evaluate the lumen and avoid potentially overestimating the stenosis percentage (Figure 2). Special attention was also directed to avoid underestimating the stenosis percentage on CA when the residual lumen is a peripheral crescent, which can happen depending on the CA view angle (Figure 3 ).

\subsection{Data Analysis}

Since the percent stenosis from angiography did not satisfy the normality 


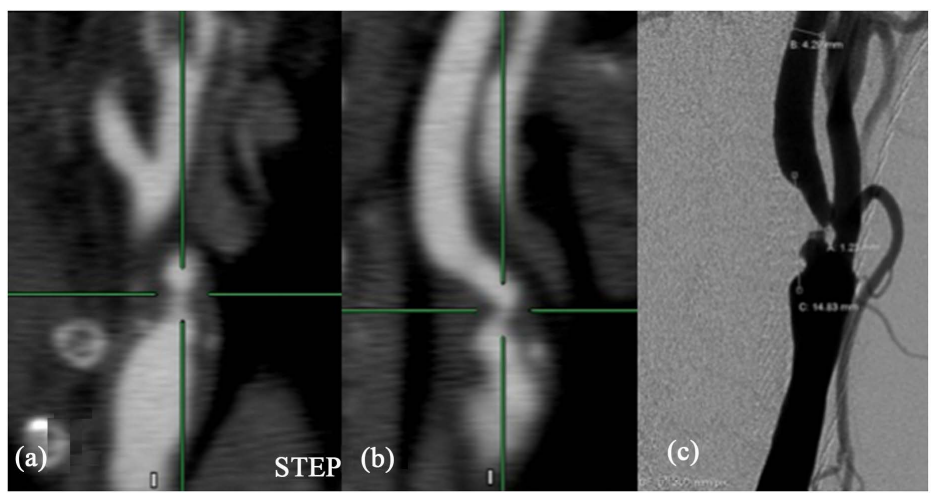

Figure 1. Illustration of creating the CTA curved sagittal thin slice MPR reformats to mirror the vessel orientation on CA using the VOXAR-3D software. (a) Coronal MPR for orientation; (b) The curved sagittal MPR mirroring the vessel in C; (c) The CA image from the same patient.

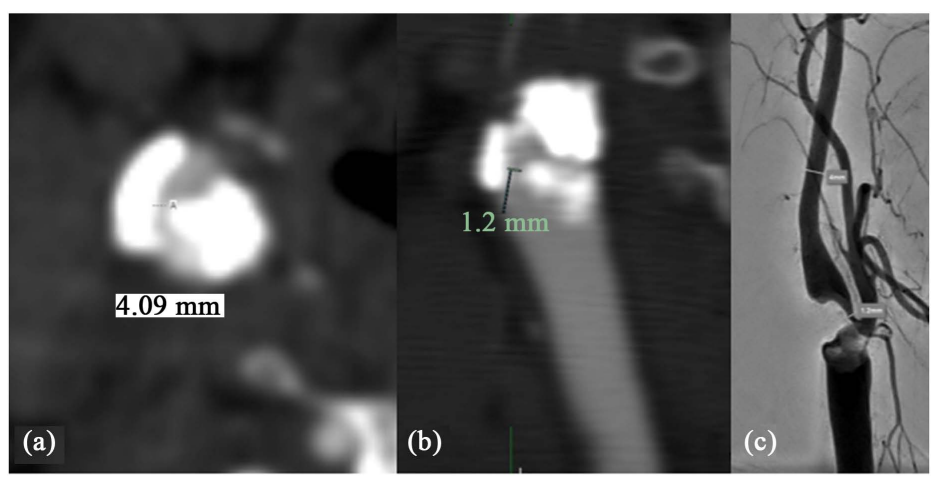

Figure 2. (a) axial CTA; (b) curved sagittal MPR CTA and (c) CA. Special attention was directed to the more densely calcified plaques with special windowing to avoid potentially overestimating the degree of stenosis. Also, notice that the stenotic segment measurement on the curved sagittal MPR created to mirror the CA images correlates better with the CA measurement.

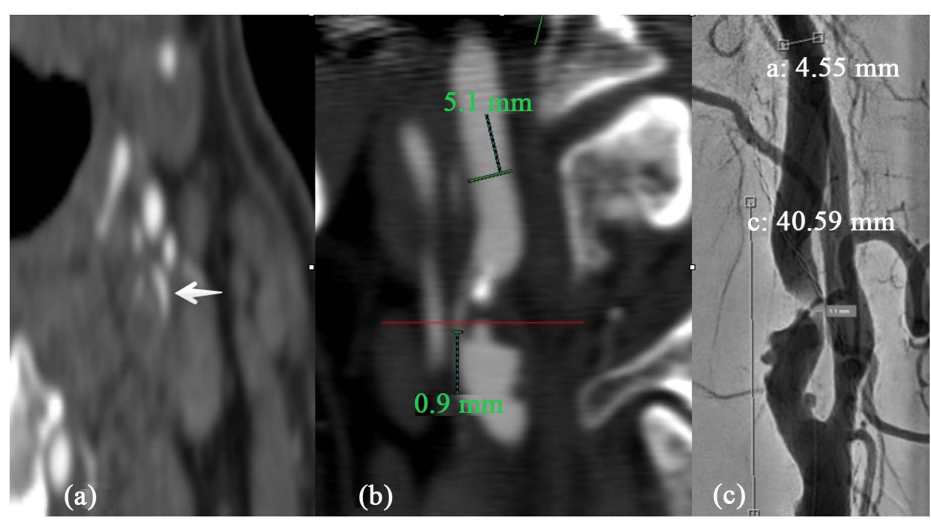

Figure 3. (a) axial CTA; (b) curved sagittal MPR CTA and (c) CA. Special attention was directed to avoid underestimating the stenosis percentage on CA when the residual lumen is a peripheral crescent (arrow). The plan of the axial image on the curved sagittal MPR is the "horizontal line". Notice the projection dependent slight underestimation of the stenotic lumen diameter on the CA image due to the crescent shaped residual lumen. The curved sagittal MPR CTA correlated better with CA. 
assumption ( $p<0.003$, Shapiro-Wilks test), the nonparametric Friedman's test was used to determine if the percent stenosis estimates differed between angiography, axial CTA and curved sagittal CTA. This was followed by post hoc Dunn's test for pairwise comparison.

\section{Results}

The percent stenosis estimates from angiography, axial CTA and curved sagittal CTA statistically differed ( $p<0.0001$, Friedman's test). Dunn's post hoc tests was used to determine whether indicate that there was a statistically significant difference between angiography and axial CTA $(p<0.0001)$, but NOT between angiography and curved sagittal CTA $(p>0.99)$. Axial CTA overestimated the percent stenosis compared to angiography (median: 69.7\% vs. 65.1\%; Figure 4).

Friedman's test indicated that the lumen diameter measured distal to stenosis statistically differed between angiography, curved sagittal CTA and axial CTA ( $p$ $<0.0001)$. Post hoc Dunn's test indicated that the difference between angiography and curved sagittal CTA was not statistically significant, but marginal ( $p=$ 0.0512 ), whereas the difference between angiography and axial CTA was statistically significant $(p=0.0005)$. Figure $4(a)$ shows the lumen diameter measured distal to stenosis is higher with axial CTA compared to angiography (median: 4.9 vs. $4.3 \mathrm{~mm}$ ). Friedman's test indicated that the lumen diameter measured at stenosis site statistically differed between angiography, curved sagittal CTA and

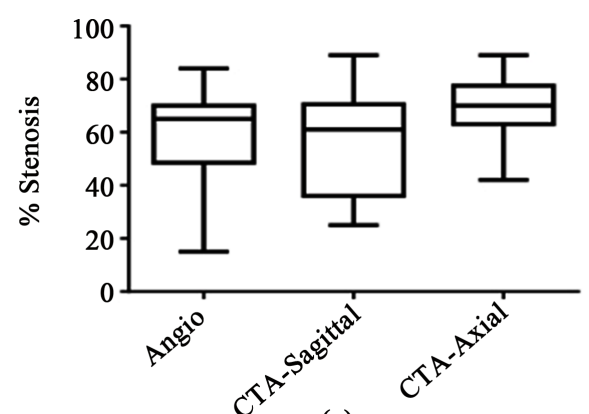

(a)

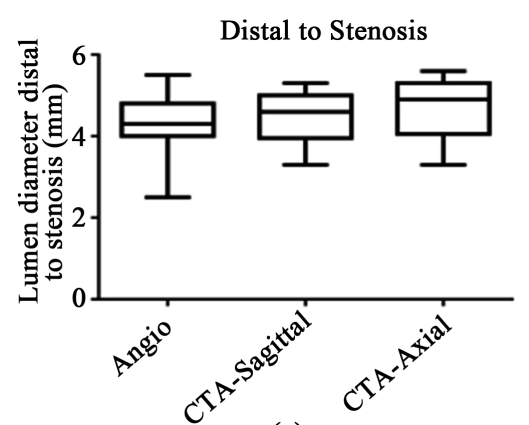

(c)
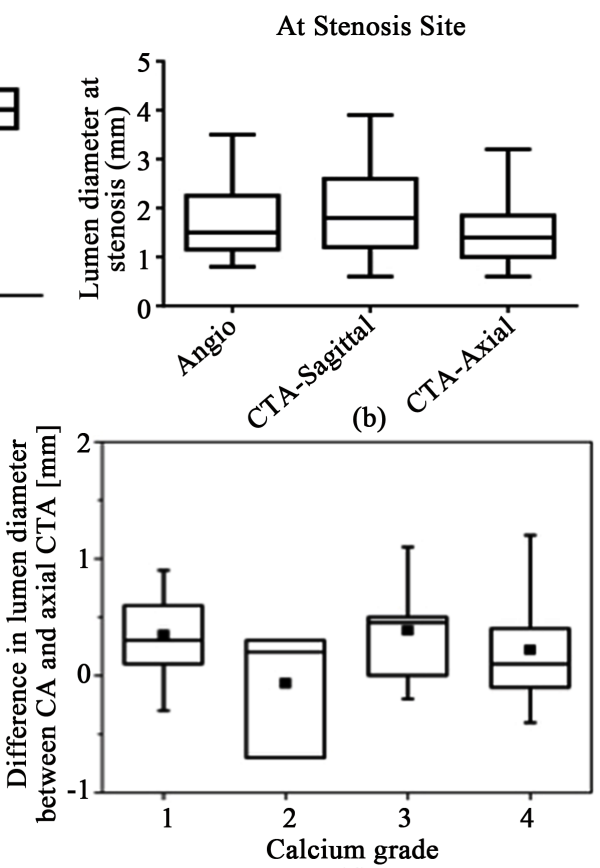

(d)

Figure 4. Box-plot of stenosis (a); lumen diameter at stenosis (b) and lumen diameter distal to stenosis (c) from CA, sagittal CTA and axial CTA. The 3 estimates statistically differed ( $p<0.001$, Friedman's test). Post hoc Dunn's test indicates that axial CTA differed from CA, but sagittal CTA did no differ from CA; (d) Difference in lumen diameter at stenosis between CA and axial CTA did not depend on calcium grade $(p=0.484)$. 
axial CTA $(p<0.0001)$. Post hoc Dunn's test indicated that the difference between angiography and curved sagittal CTA was not statistically significant ( $p=$ 0.262 ), whereas the difference between angiography and axial CTA was statistically significant $(p=0.014)$. Figure $4(\mathrm{~b})$ shows the lumen diameter measured at stenosis site is lower with axial CTA compared to angiography (median: 1.4 vs. $1.5 \mathrm{~mm}$ ). So, with respect to angiography, axial CTA overestimates lumen diameter at location distal to stenosis and underestimates lumen diameter at stenosis site contributing to overestimation of percent stenosis observed in Figure 4.

The difference in lumen diameter at stenosis site between angiography and axial CTA satisfied the normality assumption ( $p=0.943$, Shapiro-Wilks test). ANOVA indicated that the difference in lumen diameter measured at stenosis site between angiography and axial CTA did NOT statistically depend on calcium grading $(p=0.484)$. This indicated that there is insufficient evidence to support that the beam hardening effects associated with calcium content contributes to the difference in lumen diameter at stenosis site between angiography and axial CTA.

To evaluate if the relatively poorer spatiotemporal resolution of CTA contributes to reduced precision in measuring lumen diameter, we assumed the lumen diameter from angiography as the reference standard, the absolute ratio was quantified as: Precision $=($ D-DSA - D-CTA $) / D S A$, where D-DSA and D-CTA are the lumen diameters measured from angiography and CTA, respectively [11]. Linear regression of precision with DSA shows a trend towards zero with increasing D-DSA. The overall model was statistically significant $(p<0.0001)$ and D-DSA was significant predictor of precision $(p<0.0001)$. This indicates that the relatively poorer spatiotemporal resolution of CTA contributes to reduce precision in measuring lumen diameter and the effect is dependent on lumen diameter.

\section{Discussion}

Decreased arterial distensibility of a diseased vessel as a risk factor for coronary vascular disease (CVD) is well studied in the cardiology literature [1]. Functional impairment of the arterial wall may occur earlier than the structural abnormalities of the vessel wall [2]. The same observations applied to the coronary arteries can be applied to the carotid arteries in the neck. The decreased carotid distensibility in carotid atherosclerotic disease is expected to affect the blood flow and blood volume under normal physiologic condition, which is the case on CT angiography [12]. However, iatrogenic hand injection of contrast material on conventional angiography is expected to distend the vessel beyond what is expected under physiologic conditions secondary to the increase in pressure according to the Hagen-Poiseuille equation $\left(Q=\Delta P \cdot \pi \cdot r^{4} / 8 \mu L\right.$, where $Q=$ Flow, $\Delta P=$ Pressure loss, $\pi=$ Mathematical constant $\mathrm{Pi}, r=$ Radius of pipe, $\mu=$ Dynamic viscosity and $L=$ Length of pipe) [3]. A number of publications compared the percent carotid stenosis between CTA and CA, quantified the degree of stenosis 
according to the NASCET criteria on CTA [4] [5] [6] [13]. Others, additionally evaluated the relation between the volume of calcification in the mural plaques and the degree of vascular stenosis [7]. However, to our knowledge this is the first literature work evaluating the exact relationship between CTA and CA measurement at the stenotic segment hypothesizing distensibility induced underestimation of the degree of stenosis secondary to hand injection induced vascular distensibility on CA. Despite being theoretically plausible, our data showed insufficient evidence that intra-arterial hand-injection during CA leads to vascular dilation that is significant enough to affect the percentage of vascular stenosis. One factor that might have influenced our result is the small cohort of patients; a larger number may show statistical significance.

Another factor is the inherent lower resolution of CTA in comparison to CA. However, CT angiography has evolved along with the technologic advances of CT hardware and software. Modern CT angiography, performed with multidetector high-speed CT hardware, accurately and reliably depicts carotid disease and allows for direct quantification of carotid stenosis in millimeters [14] [15]. The accuracy of CTA for detection of severe carotid artery disease (70\% to $90 \%$ stenosis), specifically carotid occlusions, was shown to be very comparable to the accuracy of CA; something that our data correlated well with [9] [16]. The combined use of duplex scanning and CTA has also been found to be accurate in the diagnosis of carotid occlusion and can replace angiography in cases of carotid occlusion [17] [18]. Conversely, CTA can be less accurate than CA in distinguishing between moderate $(50 \%-69 \%)$ and severe $(70 \%-90 \%)$ carotid stenosis which is related to several factors including the inherently lower resolution of CTA in comparison to CA, different orientation of the carotid artery in relation to the imaging plane on CTA and CA and potential artifacts from soft tissue and mural plaques calcium at the site of stenosis [6] [19]. Additionally, calculating percent carotid stenosis as per NASCET can be challenging as the luminal diameter distal to the stenosis is usually reduced secondary to decreased pulse pressure distal to the stenosis which leads to collapse of the vessel wall [20] [21] [22].

Another factor that might affect vascular distensibility on both conventional and carotid angiography is the calcium content of the atherosclerotic mural plaques. As mentioned, prior scholarly work focused on correlating the volume of calcifications in the mural plaques to the degree of stenosis and showed positive results. Nonetheless, applying such technique to the daily practice of neuroradiology is time consuming, cumbersome and requires software capabilities that might not be available at every institution. For our study, we propose a new calcium grading system that can be used at any workstation. Our data showed that this calcium grading correlates with the degree of stenosis on both CTA and CA. However, the calcium scoring does not affect the percentage of stenosis when compared between CA and CTA. This could in part be related to the continued advancement in CT technology with improved spatial resolution and data post-processing [14]. Our calcium scoring consisted of 4 grades; grade 1 is $<1$ 
$\mathrm{mm}$ thick calcification regardless of circumferential involvement, grade 2 is 1 $\mathrm{mm} \leq$ thick $<1.9 \mathrm{~mm}$ and $\leq 180$ degree, grade 3 is $1 \mathrm{~mm} \leq$ thick $<1.9 \mathrm{~mm}$ and $>180$ degree or $2 \mathrm{~mm} \leq$ thick $<2.9 \mathrm{~mm}$ regardless of circumferential involvement and grade 4 is calcification thickness $>3 \mathrm{~mm}$ regardless of circumferential involvement (Figure 5). Special attention was directed to some of the more densely calcified plaques, with extra windowing used when needed to better evaluate the lumen and avoid potentially overestimating the stenosis percentage. Figure 6 shows the difference between the appearance of calcifications on soft tissue, bone and vascular windows. We found that vascular windows using window width in the range of 800 - 1100 and window levels of 350 - 550 will accentuate the contrast filled vascular lumen and minimize the beam hardening artifacts at the interface between the calcified plaque and the patent contrast-filled lumen.

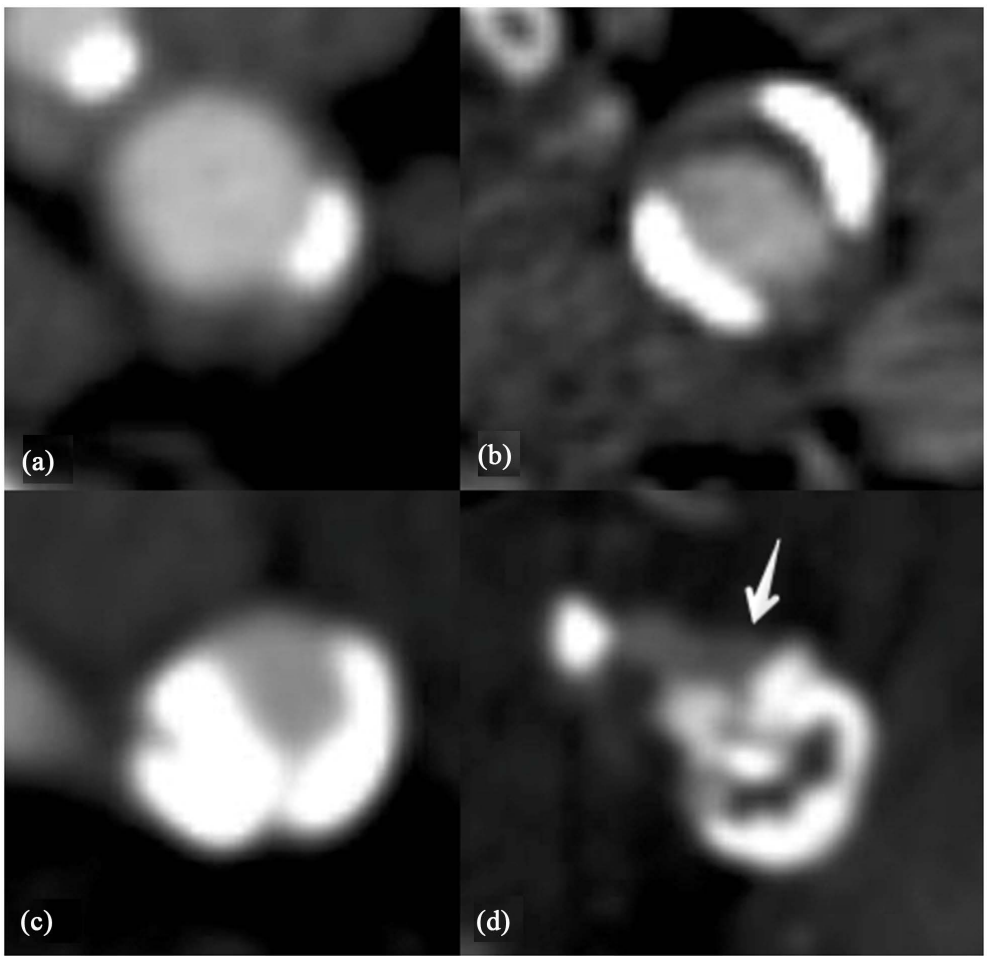

Figure 5. (a) Grade 1; (b) Grade 2; (c) Grade 3 and (d) Grade 4 calcifications. Notice the residual lumen in D (arrow).

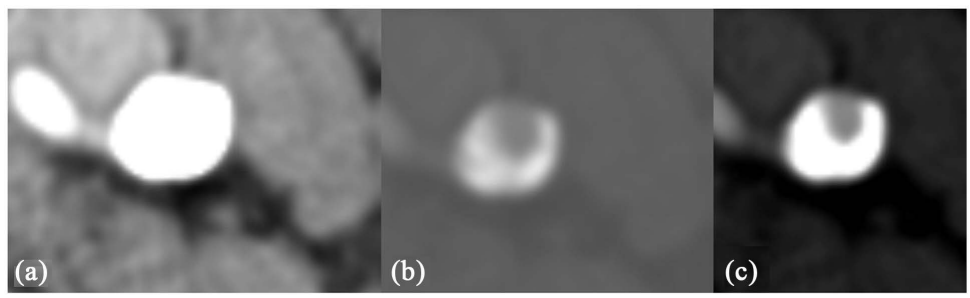

Figure 6. (a) Soft tissue window; (b) bone window and (c) vascular window. Notice the better visualization of the vascular lumen on the vascular window in comparison to the soft tissue and bone windows. 


\section{Conclusions}

At this time, there is insufficient evidence to suggest that intra-arterial hand-injection contributes to underestimation of percent stenosis during CA secondary to injection induced vessel dilatation despite larger vessel diameter at stenosis on CA in comparison to CTA in this small cohort of patients; larger cohort may show different results. Calcium grading scale correlates with the degree of vascular stenosis but does not affect the lumen diameter measurement on CTA in comparison to CA suggesting minimal or no effect of beam hardening. Importantly, curved sagittal CTA MPRs made to mirror the vascular orientation on CA should be used for measuring stenosis whenever possible as it does not differ from CA and correlated better than the axial images.

There are several limitations to our study. This is a retrospective study. Data showed insufficient evidence that intra-arterial hand-injection during CA leads to significant vascular dilation, enough to affect the percentage of vascular stenosis. One factor that might have influenced our result is the small cohort of patients; a larger number may show statistical significance.

Interobserver variability was not assessed. Nevertheless, a standardized interpretation scheme was used for analysis between carotid diameter by CTA versus intra-arterial hand injection measured on CA.

\section{References}

[1] Godia, E.C., Madhok, R., Pittman, J., Trocio, S., Ramas, R., Cabral, D., Sacco, R.L. and Rundek, T. (2007) Carotid Artery Distensibility: A Reliability Study. Journal of Ultrasound in Medicine, 26, 1157-1165. https://doi.org/10.7863/jum.2007.26.9.1157

[2] Selzer, R.H., Mack, W.J., Lee, P.L., Kwong-Fu, H. and Hodis, H.N. (2001) Improved Common Carotid Elasticity and Intima-Media Thickness Measurements from Computer Analysis of Sequential Ultrasound Frames. Atherosclerosis, 154, 185-193. https://doi.org/10.1016/S0021-9150(00)00461-5

[3] Sutera, S.P. and Skalak, R. (1993) The History of Poiseuille's Law. Annual Review of Fluid Mechanics, 25, 1-20. https://doi.org/10.1146/annurev.fl.25.010193.000245

[4] Cumming, M.J. and Morrow, I.M. (1994) Carotid Artery Stenosis: A Prospective Comparison of CT Angiography and Conventional Angiography. American Journal of Roentgenology, 163, 517-523. https://doi.org/10.2214/ajr.163.3.8079836

[5] Randoux, B., Marro, B., Koskas, F., Duyme, M., Sahel, M., Zouaoui, A. and Marsault, C. (2001) Carotid Artery Stenosis: Prospective Comparison of CT, Three-Dimensional Gadolinium-Enhanced MR, and Conventional Angiography. Radiology, 220, 179-185. https://doi.org/10.1148/radiology.220.1.r01j135179

[6] Anderson, G.B., Ashforth, R., Steinke, D.E., Ferdinandy, R. and Findlay, J.M. (2000) CT Angiography for the Detection and Characterization of Carotid Artery Bifurcation Disease. Stroke, 31, 2168-2174. https://doi.org/10.1161/01.STR.31.9.2168

[7] Alvarez-Linera, J., Benito-León, J., Escribano, J., Campollo, J. and Gesto, R. (2003) Prospective Evaluation of Carotid Artery Stenosis: Elliptic Centric Contrast-Enhanced MR Angiography and Spiral CT Angiography Compared with Digital Subtraction Angiography. American Journal of Neuroradiology, 24, 1012-1019.

[8] Fox, A.J., Eliasziw, M., Rothwell, P.M., Schmidt, M.H., Warlow, C.P. and Barnett, 
H.J.M. (2005) Identification, Prognosis, and Management of Patients with Carotid Artery near Occlusion. American Journal of Neuroradiology, 26, 2086-2094.

[9] Koelemay, M.J.W., Nederkoorn, P.J., Reitsma, J.B. and Majoie, C.B. (2004) Systematic Review of Computed Tomographic Angiography for Assessment of Carotid Artery Disease. Stroke, 35, 2306-2312. https://doi.org/10.1161/01.STR.0000141426.63959.cc

[10] Dix, J.E., Evans, A.J., Kallmes, D.F., Sobel, A.H. and Phillips, C.D. (1997) Accuracy and Precision of CT Angiography in a Model of Carotid Artery Bifurcation Stenosis. American Journal of Neuroradiology, 18, 409-415.

[11] Van den Wijngaard, I.R., Holswilder, G., van Walderveen, M.A.A., Algra, A., Wermer, M.J.H., Zaidat, O.O. and Boiten, J. (2016) Treatment and Imaging of Intracranial Atherosclerotic Stenosis: Current Perspectives and Future Directions. Brain and Behavior, 6, e00536. https://doi.org/10.1002/brb3.536

[12] Tomoto, T., Maeda, S. and Sugawara, J. (2017) Influence of Blood Flow Velocity on Arterial Distensibility of Carotid Artery in Healthy Men. The Journal of Physiological Sciences, 67, 191-196. https://doi.org/10.1007/s12576-016-0455-2

[13] Bartlett, E.S., Walters, T.D., Symons, S.P., Aviv, R.I. and Fox, A.J. (2008) Classification of Carotid Stenosis by Millimeter CT Angiography Measures: Effects of Prevalence and Gender. American Journal of Neuroradiology, 29, 1677-1683. https://doi.org/10.3174/ajnr.A1210

[14] Hu, H., He, H.D., Foley, W.D. and Fox, S.H. (2000) Four Multidetector-Row Helical CT: Image Quality and Volume Coverage Speed. Radiology, 215, 55-62. https://doi.org/10.1148/radiology.215.1.r00ap3755

[15] Chen, C.-J., Lee, T.-H., Hsu, H.-L., Tseng, Y.-C., Lin, S.-K., Wang, L.-J. and Wong, Y.-C. (2004) Multi-Slice CT Angiography in Diagnosing Total versus Near Occlusions of the Internal Carotid Artery: Comparison with Catheter Angiography. Stroke, 35, 83-85. https://doi.org/10.1161/01.STR.0000106139.38566.B2

[16] Lev, M.H., Romero, J.M., Goodman, D.N.F., Bagga, R., Kim, H.Y.K., Clerk, N.A., Ackerman, R.H. and Gonzalez, R.G. (2003) Total Occlusion versus Hairline Residual Lumen of the Internal Carotid Arteries: Accuracy of Single Section Helical CT Angiography. American Journal of Neuroradiology, 24, 1123-1129.

[17] Leclerc, X., Godefroy, O., Pruvo, J.P. and Leys, D. (1995) Computed Tomographic Angiography for the Evaluation of Carotid Artery Stenosis. Stroke, 26, 1577-1581. https://doi.org/10.1161/01.STR.26.9.1577

[18] Lubezky, N., Fajer, S., Barmeir, E. and Karmeli, R. (1998) Duplex Scanning and CT Angiography in the Diagnosis of Carotid Artery Occlusion: A Prospective Study. European Journal of Vascular and Endovascular Surgery, 16, 133-136. https://doi.org/10.1016/S1078-5884(98)80154-8

[19] Moll, R. and Dinkel, H. (2001) Value of the CT Angiography in the Diagnosis of Common Carotid Artery Bifurcation Disease: CT Angiography versus Digital Subtraction Angiography and Color Flow Doppler. European Journal of Radiology, 39, 155-162. https://doi.org/10.1016/S0720-048X(01)00302-3

[20] Silvennoinen, H.M., Ikonen, S., Soinne, L., Railo, M. and Valanne, L. (2007) CT Angiographic Analysis of Carotid Artery Stenosis: Comparison of Manual Assessment, Semiautomatic Vessel Analysis, and Digital Subtraction Angiography. American Journal of Neuroradiology, 28, 97-103.

[21] Dix, J.E., McNulty, B.J. and Kallmes, D.F. (1998) Frequency and Significance of a Small Distal ICA in Carotid Artery Stenosis. American Journal of Neuroradiology, 19, 1215-1218. 
[22] Wakhloo, A.K., Lieber, B.B., Seong, J., Sadasivan, C., Gounis, M.J., Miskolczi, L. and Sandhu, J.S. (2004) Hemodynamics of Carotid Artery Atherosclerotic Occlusive Disease. Journal of Vascular and Interventional Radiolog, 15, S111-S121.

https://doi.org/10.1097/01.RVI.0000109204.16955.84 